\title{
Discours
}

Revue de linguistique, psycholinguistique et

informatique. A journal of linguistics, psycholinguistics and computational linguistics

$20 \mid 2017$

Varia

\section{Quelques emplois discursifs des noms de parenté en général et de papa, maman, en particulier}

\section{Mathilde Salles}

\section{OpenEdition}

Journals

Édition électronique

URL : http://journals.openedition.org/discours/9259

DOI : 10.4000/discours.9259

ISSN : 1963-1723

Éditeur :

Laboratoire LATTICE, Presses universitaires de Caen

Référence électronique

Mathilde Salles, «Quelques emplois discursifs des noms de parenté en général et de papa, maman, en particulier », Discours [En ligne], 20 | 2017, mis en ligne le 22 septembre 2017, consulté le 19 avril 2019. URL : http://journals.openedition.org/discours/9259; DOI : 10.4000/discours.9259 

Revue de linguistique, psycholinguistique et informatique

\section{Quelques emplois discursifs des noms de parenté en général et de papa, maman, en particulier}

Mathilde Salles

CRISCO

Université de Caen Normandie

Mathilde Salles, «Quelques emplois discursifs des noms de parenté en général et de papa, maman, en particulier», Discours [En ligne], 20 | 2017, mis en ligne le 22 septembre 2017.

URL: http://discours.revues.org/9259 



\title{
Quelques emplois discursifs des noms de parenté en général et de papa, maman, en particulier
}

\author{
Mathilde Salles
}

CRISCO

Université de Caen Normandie

Cet article est consacré à certains fonctionnements discursifs des noms de parenté: des emplois anaphoriques avec un déterminant possessif de troisième personne (dans des anaphores possessives telles que un homme... son père) ou un article défini (dans des anaphores associatives telles que une famille... le père) et des emplois que nous appellerons «situationnels» (nous regrouperons sous ce terme à la fois leurs emplois comme termes d'adresse et les emplois - avec ou sans déterminant possessif de première ou deuxième personne - dans lesquels ils réfèrent au père, à la mère, etc. du locuteur ou de son interlocuteur).

Parmi les noms de parenté, les noms papa, maman, pépé, tata, etc., qualifiés d'«enfantins», d'«affectueux» ou de «familiers» dans les dictionnaires, connaissent surtout des emplois nus, c'est-à-dire sans déterminant. Ces emplois nus sont très diversifiés, puisque ces noms de parenté n'exigent de détermination ni dans leurs emplois situationnels ni dans leurs emplois anaphoriques. Papa désignera ainsi, selon le contexte, «mon père», «ton père», «son père » ou même «le père (d'une famille)». Une étude plus précise des emplois anaphoriques de papa, maman, etc. - de leurs emplois nus comme de leurs emplois avec un déterminant possessif ou un article défini - nous permettra de souligner les particularités de ces noms de parenté, particularités qui ne sont pas seulement de l'ordre des variations suggérées par les étiquettes d'«enfantins», d'«affectueux» ou de «familiers».

Mots clés: noms de parenté, anaphore associative, anaphore possessive, termes d'adresse, chaînes de la conversation

This paper is devoted to some discursive operations of kinship nouns: anaphoric uses with a third person possessive (in possessive anaphora such as a man... his father) or a definite article (in associative anaphora as a family... the father) and uses we call "situational", i.e. both their uses as address terms and uses - with or without a first or second person possessive - in which they refer to the speaker's or interlocutor's father, mother, etc.

Among the kinship nouns, nouns like dad, mom, grandpa, auntie, etc., described as "childish", "loving" or "familiar" in dictionaries, have mainly naked uses, i.e. without a determiner. These naked uses are various, since kinship nouns do not require a determiner neither in their situational uses nor in their anaphoric uses. Depending on the context, dad will refer to "my father", "your father", "his/her father" or even "the father (of some family)". A more specific study of anaphoric uses of dad, mom, etc. - their naked uses as well as their uses with a possessive determiner or a definite article - will allow us to emphasize the characteristics of these kinship nouns, characteristics that are not just the variations suggested by the labels "childish", of "affectionate" or "familiar".

Keywords: kinship nouns, associative anaphora, possessive anaphora, address terms, conversational chains 


\section{Introduction ${ }^{1}$}

Les noms de parenté apparaissent comme les parangons de la catégorie des noms relationnels, noms qui ont, selon Hiz (I968), un complément explicite ou tacite ${ }^{2}$ et «dont la référence virtuelle implique une mise en relation» (Milner, 1982: 28). Les noms de parenté sont, en effet, fréquemment les premiers cités dans les études sur les noms relationnels ${ }^{3}$ et leur intégration à cette catégorie, contrairement à celle d'autres noms comme les noms d'action ou de propriété (voir Huyghe, 20I5: I6), n'est guère remise en question. À l'intérieur même de leur catégorie, leur dimension relationnelle a toutefois pu être graduée: Barque (2015:37), dans une étude consacrée aux noms relationnels humains (noms de parenté, mais aussi autres «noms d'humains en relation interpersonnelle» comme ami, voisin, collègue, client...), distingue des noms «faiblement relationnels» (père, grand-père, tante...), qui, par exemple, ne nécessitent pas une spécification de l'élément en relation dans leurs emplois de statut (exemple [r] $)^{4}$, et des noms «fortement relationnels» (fils, petit-fils, søur, cousin...), qui impliquent une relation de dépendance plus forte et nécessitent une telle spécification dans ces mêmes emplois (exemple [2]):

[I] Max est père/grand-père.

[2] Max est ${ }^{*}$ fils $/ *$ petit-fils/fils de gendarme/petit-fils de gendarme.

Mais, qu'ils soient fortement ou faiblement relationnels au sens de Barque (2015), aucun d'entre eux n'exige une telle spécification dans un autre type d'emploi, leur emploi comme nom de membre, à l'œuvre dans les anaphores associatives (exemples [3a-b]):

[3a] Un couple m'a rendu visite hier; le mari était insupportable.

(Milner, 1982: 28)

1. Je remercie les deux relecteurs anonymes de la revue Discours pour l'ensemble de leurs remarques et suggestions.

2. Un complément dont la réalisation n'est pas toujours possible, comme le souligne Barker (2OII : IIII) avec le nom stranger: «despite the fact that a possessor argument is conceptually obligatory [...], it is not possible to express the possessor relatum for stranger overtly, either by means of genitive of phrase (*the stranger of John) or by a prenominal possessive (*John's stranger)».

3. Ce sont les premiers exemples de noms relationnels donnés par De Bruin et Scha (1988; sister dès le résumé, brother dans la première section) ou Nakamura et Mori (2004; mother); c'est aussi le premier type de relation nommé par Barker (2OII ; «family relations», juste avant «body parts») ou Nakamura et Mori (2004; «kinship relations», juste avant «whole-parts relations»), entre autres.

4. Les noms de parenté peuvent, à l'instar d'autres noms d'humains, relationnels ou non (noms de métier, de nationalité, de fonction), désigner des statuts, lorsqu'ils sont employés sans déterminant dans une construction attributive (voir Barque, 20I5): Max est grand-père/père/père de trois enfants/fils et petit-fils de gendarme... Comme pour les autres noms d'humains étudiés par Kupferman (I99I) et Giry-Schneider (I99I), ces emplois permettent, quand le sens du nom de parenté s'y prête, des précisions temporelles: Max est grand-père depuis bier (vs * Max est un grand-père depuis bier). 
[3b] Dans les familles d'origine immigrée notamment, la mère est en porte à faux entre sa culture d'origine et sa volonté d'intégration, elle est complètement larguée au niveau scolaire et les enfants en profitent.

(Dernières nouvelles d'Alsace; dans Kleiber, 1999: 287; 2003: 99; 2008:320)

Certains d'entre eux peuvent même se passer de la spécification de l'élément avec lequel ils entrent en relation (spécification sous forme de complément du nom ou de déterminant possessif) dans leurs emplois strictement relationnels. Il s'agit essentiellement des termes papa, maman, pépé, tata, etc., qualifiés d'«enfantins», d'«affectueux» ou de «familiers» dans les dictionnaires. Ces noms de parenté n'exigent de détermination possessive ni dans leurs emplois situationnels ${ }^{5}$ ni dans leurs emplois anaphoriques. Papa désignera ainsi, selon le contexte, «mon père», «ton père», «son père»... ou même «le père», dans une interprétation qui, contrairement aux trois précédentes, ne sera plus strictement relationnelle - une interprétation comme nom de membre comparable à celle de mari et mère dans les exemples d'anaphores associatives (voir $[3 a-b])$.

Dans les sections suivantes, nous nous attacherons aux différents emplois discursifs des noms de parenté que nous venons d'évoquer: emplois anaphoriques de père, mère, etc. dans la section 2 ; emplois situationnels de père, mère, mais aussi de papa, maman dans la section 3; emplois anaphoriques de papa, maman dans la section 4 . Nous nous appuierons sur des exemples authentiques d'origine très diverse: roman, théâtre, correspondance personnelle, forum de discussion, chanson, critique de film... L'étude des emplois anaphoriques de papa, maman nous permettra de souligner la singularité de ces termes et nous conduira à une caractérisation plus générale et - nous l'espérons - plus juste que celle apportée par les étiquettes d'«enfantins», d'«affectueux» ou de «familiers» des dictionnaires.

\section{Noms relationnels de parenté ou noms de membres dans leurs emplois anaphoriques}

\subsection{Anaphore possessive ou anaphore associative}

Les noms de parenté connaissent deux types d'emplois anaphoriques qui ont été très précisément décrits par Kleiber (1999, 2000, 2001, 2002, 2003, 2008) et dont nous ne donnerons ici qu'une présentation synthétique ${ }^{6}$ : des emplois en anaphore possessive et en anaphore associative.

5. On rassemble ici leurs emplois comme termes d'adresse, dans lesquels ils réfèrent à l'interlocuteur, et d'autres emplois, dans lesquels ils réfèrent à celui que le locuteur ou, plus rarement, son interlocuteur appelle papa, maman, pépé (voir infra, section 3).

6. Nous renvoyons aux différents travaux de Kleiber pour une analyse plus complète de ces deux relations anaphoriques et des différences de saisies référentielles opérées par le déterminant possessif et l'article défini. 
Ces emplois s'écartent des emplois anaphoriques prototypiques (les emplois de reprise ${ }^{7}$ ) dans la mesure où ils permettent l'introduction de nouvelles entités. L'introduction d'entités nouvelles fait partie de la définition même de l'anaphore associative (voir notamment Kleiber, 200I), ce qui n'est pas le cas de l'anaphore possessive qui peut ou non introduire du nouveau. Mais, pour introduire du nouveau, pour que son $X$ puisse être utilisé pour référer à une entité $\mathrm{X}$ qui n'a pas encore été mentionnée, l'anaphore possessive réclame, selon Barker (1995: Io), une relation de «possession lexicale», par exemple, une relation de parenté ou encore une relation partie-tout. Barker (1995: 10) oppose ainsi «A man walked in. His daughter was with bim», séquence dans laquelle le syntagme nominal (SN) possessif His daugther, avec le nom relationnel daugther, peut être utilisé pour introduire une nouvelle entité dans le discours, à "A man walked in. \#His firetruck was visible through the window», séquence dans laquelle le possessif His firetruck, avec un nom de possession extrinsèque sans relation habituelle avec un homme ( $v$ s un pompier), parait étrange pour une première mention de l'entité en question.

L'anaphore possessive permet donc d'introduire un nouvel individu dans le discours, en explicitant le lien de parenté («fils de» en [4a], «mari de» en [4b]) entre cet individu et un autre mentionné auparavant (le docteur Porquier en [4a], la femme dont le locuteur a été l'amant en [4b]):

[4a] [...] ce gros monsieur est le docteur Porquier, le médecin qui soigne la société de Plassans. Un homme heureux, je vous assure. Il n'a qu'un chagrin, son fils Guillaume...

(Frantext: É. Zola, La conquête de Plassans [1874], Paris, Gallimard, 1960, p. 933)

[4b] J'ai été l'amant d'une femme mariée qui venait me raconter en gémissant que son mari la trompait et qu'elle en était jalouse. Certes, le cas est rare!

(Frantext: M. Du Camp, Mémoires d'un suicidé [1853], Paris, Librairie nouvelle, 1855, p. 247)

L'anaphore associative, avec laquelle l'introduction de nouvelles entités se fait sous la forme de SN définis incomplets (le $N)$, n'autorise les noms de parenté que si ceux-ci sont en outre présentés comme des membres d'une collection (une famille, un couple). L'article défini ne suffit en effet pas à assurer l'introduction du fils ou du mari en $[5 \mathrm{a}-\mathrm{b}]$, si le seul lien avec le contexte antécédent est un lien de parenté («fils du docteur Porquier», «mari de la femme dont j'ai été l'amant»), alors que la relation «membre de» qui relie les noms collectifs couple, famille aux noms de

7. Emplois que nous n'étudierons pas ici, mais que les noms de parenté permettent bien évidemment aussi (par exemple: «Il résolvait avec une facilité déconcertante les devinettes: Trois Russes ont un frère. Ce frère meurt sans laisser de frères. Comment est-ce possible?»; Frantext: G. Perec, La vie mode d'emploi: romans [1978], Paris, Le livre de poche, 1995, p. 508). 
membres mari, mère, enfants, permet ce type d'introduction (exemples [6a-b]) et exclut, à l'inverse, le possessif (exemples $[7 a-b])^{8}$ :

[5a] [...] ce gros monsieur est le docteur Porquier, le médecin qui soigne la société de Plassans. Un homme heureux, je vous assure. Il n'a qu'un chagrin, ?le fils Guillaume...

[5b] J'ai été l'amant d'une femme mariée qui venait me raconter en gémissant que ?le mari la trompait et qu'elle en était jalouse.

[6a] Un couple m'a rendu visite hier; le mari était insupportable.

[6b] Dans les familles d'origine immigrée notamment, la mère est en porte à faux entre sa culture d'origine et sa volonté d'intégration, elle est complètement larguée au niveau scolaire et les enfants en profitent.

[7a] Un couple m'a rendu visite hier; *son mari [= du couple] était insupportable.

[7b] Dans les familles d'origine immigrée notamment, *leur mère [= des familles $]$ est en porte à faux entre sa culture d'origine et sa volonté d'intégration, elle est complètement larguée au niveau scolaire et *leurs enfants [= des familles] en profitent.

\subsection{Contextes favorables aux deux modes anaphoriques}

Enfin, lorsque la relation «membre de» se double d'un lien de parenté direct avec un individu mentionné dans le contexte, les deux modes anaphoriques - anaphore associative et anaphore possessive - deviennent possibles, le choix de l'un ou l'autre soulignant soit l'appartenance au groupe, soit le lien de parenté (voir Kleiber, 2002 et 2003):

[8a] Hier, la cour d'assises du Haut-Rhin a consacré une première journée à cerner la personnalité de l'accusée. [...] À la maison, le frère aîné faisait régner un ordre quasi totalitaire... Quant à la mère, elle s'inquiétait de ce que sa fille «buvait trop de Coca-Cola».

(Dernières nouvelles d'Alsace; dans Kleiber, 2002: 234; 2003: 108)

[8b] Hier, la cour d'assises du Haut-Rhin a consacré une première journée à cerner la personnalité de l'accusée. [...] À la maison, son frère aîné faisait régner un ordre quasi totalitaire... Quant à sa mère, elle s'inquiétait de ce que sa fille «buvait trop de Coca-Cola».

8. Selon Kleiber (2000: 72), si le possessif ne convient pas en [7a-b], c'est parce que «mari et mère sont des termes relationnels, mais la relation fonctionnelle qui les caractérise sémantiquement [et qui offre ici la relation de dépendance nécessaire à l'emploi du possessif] les unit non pas à famille ou couple, mais à enfants pour mère et femme pour mari». 
[9a] Elle appartenait à une famille modeste (son père était maître d'école) et trouva difficilement à se marier en raison d'une infirmité qui la défigurait, tout comme Prue, l'héroïne de Sarn.

(Frantext: M. Webb, Sarn, J. de Lacretelle et M. T. Guéritte (trad. fr.) [1930], Paris, Grasset, 1999, p. 7)

[9b] Elle appartenait à une famille modeste (le père était maître d'école) et trouva difficilement à se marier en raison d'une infirmité qui la défigurait, tout comme Prue, l'héroïne de Sarn.

Dans l'exemple [8a], analysé par Kleiber (2002 et 2003), c'est d'abord la première option qui a été choisie ${ }^{9}$ (le frère aîné, la mère), de façon à décrire la famille dont l'accusée fait partie, avant l'option parenté directe (sa fille), choisie en revanche pour rapporter les inquiétudes de la mère pour sa fille. L'option parenté directe (son père) est aussi choisie en [9a] pour laisser la place centrale au personnage féminin, elle.

Il n'est pas nécessaire que le nom collectif lui-même soit mentionné pour que la dimension «membre de» du nom de parenté soit activée et, ainsi, que l'anaphore associative puisse s'établir. L'exemple [8a] proposé par Kleiber, avec la simple mention à la maison, le montre déjà. D'autres exemples, tels ceux de Milner (1982) et de Kleiber (200I, 2002 et 2003), le montrent encore:

[Ioa] On m’a présenté une jeune fille à marier, mais la mère était impossible.

(Milner, 1982: 27)

[rob] Un beau mariage a eu lieu hier à Pfaffenheim. Les mariés étaient en blanc. Les parents étaient ravis.

(Kleiber, 200I: 356)

La mention d'un mariage permet l'introduction des noms de parenté mère, parents, sous l'angle non de leur relation de parenté directe avec la jeune fille ou les mariés (ce qui aurait été le cas si le possessif avait été utilisé), mais sous l'angle de leur rôle dans un ensemble ou encore, comme l'écrit Kleiber (2001 : 357), de leur "place dans un ensemble collectif plus vaste, la famille, qui regroupe d'autres membres». L'anaphore associative gomme alors certaines relations interpersonnelles, pour souligner une place dans la famille. Le jeu des 7 familles ne pourrait pas fonctionner autrement: dans la famille $X$, je demande le père (qui est aussi le fils ou le gendre du grand-père, le mari de la mère...) / la fille (qui est aussi la petite-fille du grand-père, la sœur du fils...)/le grand-père (qui est aussi le père ou le beau-père du père, le mari de la grand-mère...), etc.

On voit ici l'incidence de ces modes anaphoriques - l'anaphore possessive d'une part, l'anaphore associative d'autre part - sur l'interprétation relationnelle des noms de parenté: interprétation strictement relationnelle lorsque la place du complément

9. Voir «le révélateur à la maison», souligné par Kleiber (2003: 108). 
est saturée par un possessif (son fils = «le fils de lui/ elle», son référant nécessairement à un individu $\left.{ }^{10}\right)$, moins strictement relationnelle lorsque cette même place n'est pas saturée (le père = celui qui occupe la place de père dans un ensemble, mais qui, dans les relations interpersonnelles à l'intérieur de cet ensemble, pourra être à la fois un père, un mari, un fils...). La relation sémantique qui fonde l'anaphore associative est une relation d'inclusion, la relation membre-collection, et, généralement, il s'agit d'une relation asymétrique dans laquelle c'est le nom de collection (régiment, forêt, etc.) qui est syncatégorématique ${ }^{11}$ et non le nom de membre (soldats, arbres, etc.). Le membre, entité autonome, n'est en effet pas défini par sa relation, contingente, à la collection, alors que la collection, elle, est définie par rapport aux éléments qui la composent. Comme il a souvent été noté ${ }^{12}$, cette orientation de la collection aux membres différencie d'ailleurs fondamentalement la relation membre-collection de la relation partie-tout au sens strict. Il y a asymétrie dans les deux cas, mais l'orientation ne se fait pas dans le même sens, de la collection vers les membres, dans un cas, de la partie vers le tout, dans l'autre: alors qu'un arbre n'est défini ni par ses parties, ni par son éventuelle appartenance à un ensemble plus vaste, les parties de l'arbre seront, elles, définies comme telles (un tronc se définit comme une partie d'un arbre) et une forêt sera définie comme un ensemble d'arbres.

\section{Emplois d'adresse et emplois dans les chaînes de la conversation}

\subsection{Emplois situationnels: des formes de noms propres}

Outre ces emplois anaphoriques, certains noms de parenté connaissent des emplois nus, sans déterminant, référant à l'interlocuteur lorsqu'ils sont employés comme termes d'adresse ou référant à celui que le locuteur ou son interlocuteur appelle père, grand-père ou papa, pépé. Le nom de parenté devient une sorte de nom propre qui, toutefois, ne s'affranchit pas de fortes contraintes égocentriques: je peux employer papa pour parler de mon propre père et, quelquefois, de celui de mon interlocuteur (par exemple, si je suis la mère de ce dernier), en d'autres termes, je peux désigner ainsi celui qui s'appelle papa pour moi et/ou pour toi, celui que toi et moi avons l'habitude d'appeler papa. En revanche, si je suis étranger à une famille, je ne dirai probablement pas à un enfant «Est-ce que papa est à la maison?» pour parler de son père, j'emploierai plus volontiers ton papa ou ton père. Papa, maman sont ainsi des formes de noms propres liés à la sphère familiale (voir Cornulier, 200I : 22), des noms propres dont la convention d'attribution peut être décrite de la manière suivante:

10. Un complément prépositionnel permettrait aussi, outre la référence à un individu - fils de Max - la référence à une collection - fils de la famille: «[...] cette maison décrépite avait formé à son image le dernier fils de la famille» (Frantext: M. Yourcenar, Denier du rêve [1959], Paris, Gallimard, I99I, p. I9I); «Surtout lui, le fils de la famille» (Frantext: B. Bayon, Le lycéen, Paris, Quai Voltaire, 1987, p. I89).

11. On reprend ici la caractérisation de Kleiber (200I : 243), pour qui les noms syncatégorématiques sont ceux qui désignent des entités manifestant «une dépendance ontologique vis-à-vis d'autres occurrences».

12. Voir notamment Lecolle (1998), Villar (2006) et Joosten (2010). 
Convention d'attribution du nom propre maman: À l'intérieur de la cellule familiale, dans une communication impliquant les enfants, la mère (et elle seule) est ou peut être désignée sous le nom de maman.

(Cornulier, 200I : 22)

Les termes Madame, Monsieur connaissent des emplois comparables: à la fois termes d'adresse (Monsieur en [II]) et formes de noms propres «égocentriques» désignant la maîtresse ou le maître du locuteur ou de son interlocuteur (Madame en [II], celle qui s'appelle Madame pour la vieille bonne, d'abord interlocutrice de «Madame est-elle rentrée?», puis locutrice de «Depuis quand Monsieur a-t-il vu Madame rentrer pour six heures et demie?»):

[II] Au second étage, il sonna.

Une vieille bonne qui l'avait élevé, une de ces servantes maitresses qui sont les tyrans des familles, vint ouvrir; et il demanda avec angoisse:

- Madame est-elle rentrée?

La domestique haussa les épaules:

- Depuis quand Monsieur a-t-il vu Madame rentrer pour six heures et demie?

(G. de Maupassant, «Monsieur Parent», in Le Horla et autres contes cruels et fantastiques, Paris, Garnier, 1989, p. 310-311)

Bien qu'on ne soit généralement pas appelé papa, Madame sans répondre à des propriétés précises ${ }^{13}$, un certain nombre de caractéristiques rapprochent papa, maman, Madame, Monsieur de noms propres dans ces différents emplois nus:

- la majuscule de Madame, Monsieur ou encore celle de Père et Maman dans les exemples $[\mathbf{I} 2 \mathrm{a}-\mathrm{b}]$ :

[I2a] J'ai parlé à Père au petit déjeuner de la possibilité de construire un solarium attenant à la salle à manger pour disposer au moins d'une pièce lumineuse et moderne.

(A. Munro, «Pouvoirs», in Fugitives: nouvelles, J. Huet et J.-P. Carasso (trad. fr.), Paris, Points, 2009, p. 3II)

[I2b] D'un seul coup, j'ai eu de la peine pour Maman; beaucoup de peine, tant de peine, j’ai pris sa tête dans mes mains, je l'ai serrée, je ne pouvais plus la lâcher. Et Maman n'arrêtait pas de me caresser et de pleurer.

(A. Tchekhov, La cerisaie, E. Pavis-Zahradnikova et P. Pavis (trad. fr.), Paris, Le livre de poche, 2016, p. 30)

- la possibilité de constituer un $\mathrm{SN}$ sans déterminant dans toutes les positions syntaxiques (Papa est-il rentré?, Demande à papa...);

13. Généralement seulement car, comme le note Wilmet (2016:20), si «Maman, Grand-Père [que Wilmet traite comme des antonomases de noms communs en noms propres] peuvent fort bien traduire une ascendance familiale du premier ou du second degré, [...] on connaît des maris qui appellent leur femme "Maman" et un de [ses] amis avait affublé son chat - exceptionnellement placide, il est vrai - du nom de "Pépé”». 
- l'autonomie et l'unicité référentielle, puisque, comme les noms propres, papa, maman, «suffisent à instancier un particulier» (Charolles, 2002: 247) dans un contexte donné. Ils constituent un nom-«name» pour un ou des locuteurs particuliers ${ }^{14}$.

\subsection{Les chaînes de la conversation}

Mais les emplois de papa, maman ne sont pas strictement identiques à ceux de noms propres ordinaires ni même à ceux de Madame, Monsieur, dans les «chaînes de la conversation» (Corblin, 2005). Ils ne le sont pas non plus à ceux de SN possessifs comme mon père, ton père. La question de leur emploi ne se pose pas tout à fait dans les mêmes termes: l'appartenance à une communauté épistémique très restreinte garantit ce qu'on pourrait appeler la réciprocité désignative (c'est-à-dire le fait que locuteur et interlocuteur puissent utiliser le même désignateur, par exemple papa, pour parler d'un individu), mais l'appartenance à une communauté épistémique plus large rend encore possible l'emploi de ces noms par le seul locuteur.

Le concept de communauté épistémique ${ }^{15}$ permet à Corblin (2005) d'expliquer le choix référentiel dans les chaînes conversationnelles, chaînes d'une grande pauvreté lorsqu'on les compare à celles des "discours à interlocuteur générique» (Corblin, 2005: I). Corblin oppose ainsi la conversation aux autres discours:

Le paramètre clé pour définir ce que j’appelle ici conversation est que du langage est échangé entre deux protagonistes concrets, définis par un système réel de communautés épistémiques. A s'adresse à $\mathrm{B}$ et ils s'identifient mutuellement comme appartenant à un certain nombre de communautés constituées par des connaissances/croyances partagées. [...] J'opposerai la conversation à ce que j'appelle les discours à interlocuteur générique. Dans ce cas, le discours s'adresse à un interlocuteur virtuel, dont le rôle est susceptible d'être assumé par une pluralité d'individus concrets. De ce fait, celui qui le tient ne peut faire fond sur la reconnaissance de communautés épistémiques réelles qui relieraient les protagonistes du discours.

(Corblin, 2005: I)

Si locuteur et interlocuteur appartiennent à une communauté épistémique dans laquelle le nom propre Pierre a cours pour désigner Pierre, alors ce nom propre n'est pas seulement un désignateur nominal possible, c'est le seul possible. La chaîne conversationnelle référant à Pierre sera formée uniquement d'occurrences de ce nom propre et de pronoms:

14. Même s'ils ne sont pas le «vrai» nom de l'individu, comme le souligne Ryan lorsqu'il rencontre son père, dans la série britannique Happy Valley (saison I, épisode 7):

Tommy: I am your dad, Ryan. [...]

Ryan: What's your name? [long silence]

Tommy: Call me dad.

Ryan: I mean your real name.

Tommy: Ok, well, it's Tommy.

15. Emprunté à Beyssade (I994 et 1998) et défini par Corblin (2005: 5) comme «des ensembles d'individus unifiés par des connaissances ou croyances». 
Si votre fils s'appelle Pierre, et si vous êtes dans une conversation avec un interlocuteur pour lequel ce NP est en usage, donc un interlocuteur appartenant à $\mathrm{CE}_{\text {Pierre }}{ }^{16}$ il n'y aura pas d'autre désignateur possible que Pierre et $i l$. Cela implique notamment que mon fils ne sera pas alors un désignateur possible.

(Corblin, 2005:6)

Si locuteur et interlocuteur n'appartiennent pas à une telle communauté épistémique, un autre désignateur sera utilisé, par exemple mon fils, mon père. Et, dans cette autre communauté épistémique, ce désignateur apparaîtra aussi comme un «désignateur propre» ${ }^{17}$ et comme le seul utilisable. Si je parle de mon père en disant mon père, mon interlocuteur en parlera en disant ton père (ou, évidemment, il), mais non le type, le professeur, le mari de ta mère. Mais le fait que je parle de mon père en disant papa ne permet pas forcément à mon interlocuteur d'utiliser ce même désignateur. La communauté épistémique qui rend possible une telle réciprocité désignative est très restreinte (mes frères et sœurs et moi, éventuellement ma mère) et je peux finalement utiliser papa pour parler de mon père avec un interlocuteur qui n'appartient pas à la $\mathrm{CE}_{\text {papa }}$, mais à une autre $\mathrm{CE}$, une $\mathrm{CE}$ à double désignateur papa/ton père - une $\mathrm{CE}_{\text {papa/ton père. }}$ C'est ce qui se produit lorsque je parle de mon père à ma tante, qui, elle, me parle de son propre père en disant papa $\left(\mathrm{CE}_{\mathrm{papa} / \mathrm{pépe} e}\right) \ldots$ Cette dernière $\mathrm{CE}$ est souvent, elle aussi, très restreinte : la plupart des locuteurs n'emploient papa pour parler de leur père qu'avec des proches, l'emploi d'un tel désignateur dans une CE plus large étant souvent ressenti comme infantile.

L'absence possible de réciprocité désignative distingue ainsi les désignateurs papa, maman des autres désignateurs dans les chaînes de la conversation. Si un locuteur emploie Madame pour parler d'un tiers, son interlocuteur emploiera ce même désignateur (voir l'exemple [II]); la CE peut là aussi être très restreinte, mais c'est une $\mathrm{CE}_{\mathrm{Madame}}$. Alors qu'avec papa, on trouve à la fois des $\mathrm{CE}_{\text {papa }}$ et des $\mathrm{CE}_{\mathrm{papa} / \text { ton }}$ père et, ainsi, des chaînes conversationnelles un peu moins pauvres que les autres. Ce n'est toutefois pas là la seule particularité de ces noms de parenté.

\section{Papa, maman... des noms de parenté pas comme les autres}

\subsection{Deux séries de termes en français?}

Selon Aikhenvald (2013: 16), nombre de langues distinguent, parmi les noms de parenté, les termes d'adresse des autres termes (les «reference terms» chez Aikhenvald), les premiers ne pouvant généralement pas être possédés (c'est le cas, précise Aikhenvald, dans certaines langues arawakiennes - nanti, tariana - et dans beaucoup de langues tucanoanes orientales). En français, certains noms de

16. $\mathrm{CE}=$ communauté épistémique.

17. Selon Corblin (2005: 7), «[...] dans une communauté épistémique, il faut qu'une chose [ou un individu] ait un nom : parmi l'ensemble des descriptions qui s'appliquent à un lieu ou à un objet [ou à un individu], l'une d'entre elles est distinguée, que nous avons appelée son "désignateur propre”». 
parenté sont plus volontiers termes d'adresse, d'autres plus volontiers «termes référentiels». Les termes qualifiés d'«enfantins», d'«affectueux» ou de «familiers» dans les dictionnaires, comme papa, maman, pépé, tata, appartiennent à la première catégorie: ils sont plus fréquemment employés comme termes d'adresse que les autres noms de parenté et, s'ils n'excluent pas la détermination possessive, connaissent beaucoup plus d'emplois nus.

En emploi nu, ils autorisent en fait les mêmes types d'emplois «référentiels» que les autres noms de parenté, non seulement les emplois déjà évoqués dans les chaines de la conversation, mais aussi des emplois comparables aux anaphores possessives (exemples [4a-b]), comme en [I3] et [14], et des emplois comparables aux anaphores associatives (exemples [6a-b]), comme en [is] et [16], après le nom collectif famille:

[13] Papa est dans la finance et a fait tourner son gros carnet d'adresses, ce type n'a aucun talent. Comparativement, Elie Semoun a lui au moins un talent relatif.

(forum jeuxvideo.com, consacré à Kev Adams; l'énoncé est à propos de Kev Adams et $p a p a=\ll$ son père»)

[r4] Qu'il se soit payé l'ancien appartement de Claude Zidi, soit... Papa est richissime, mais qu'il ne paye pas d'impôt!!! Là non, STOP!

(forum bfmtv.com, consacré à l'appartement à 7 millions d'euros de Thomas Fabius; l'énoncé est à propos de Thomas Fabius et papa $=$ «son père»)

[i5] Une famille des plus charmantes

Trois enfants maman papa

Partit un beau jour de Nantes

Pour visiter le Canada

(Frantext: C. Trenet, «Voyage au Canada», in Chansons (1946-1959), Paris, Le livre de poche, 1993, p. 235)

[16] Pas loin dans une clairière, y a une famille qui casse la croûte, avec table et pliants... Papa, maman et la grande fille... Mignons comme tout... Qui feraient pas de mal à une mouche...

(Frantext: B. Blier, Les valseuses [1972], Paris, J’ai lu, 1989, p. 417)

Dans ces emplois anaphoriques ${ }^{18}$, le lien de parenté avec un autre référent peut s'établir en l'absence de toute marque relationnelle (exemples [13]-[I4], sans détermination possessive) ou l'extraction d'un membre de la collection peut se faire en l'absence de toute détermination nominale (exemples [I5]-[I6], sans l'article défini qu'on retrouve en revanche avec le nom fille - la grande fille - en [16]). Ces emplois nus se trouvent essentiellement dans des formes de communication, orales ou écrites,

18. Anaphoriques si l'on estime que leur interprétation référentielle est, comme pour les anaphores possessives (exemples $[4 a-b]$ ) et les anaphores associatives (exemples [6a-b]), liée au contexte linguistique. 
manifestant une certaine "proximité» ${ }^{19}$ : forums de discussion (exemples [I3]-[I4]), chanson (exemple $\left[{ }_{15}\right]$ ), roman au «style parlé» (exemple [i6])...

Les emplois de le papa, la maman (avec, cette fois, utilisation de l'article défini) en anaphore associative ou de son papa, sa maman en anaphore possessive semblent eux aussi caractéristiques d'une communication de proximité, correspondance personnelle et conversation spontanée rapportée dans les exemples d'anaphores associatives en [I7] et [I8], correspondance personnelle et critique de film ${ }^{20}$ dans les exemples d'anaphores possessives en [19] et [20]:

[I7] Tu peux dire au capitaine D’Arpentigny que la famille Fouet est dans les honneurs et la fortune. Le papa est conseiller à la cour d'appel, le fils substitut, et on vient d'épouser 60000 francs de rentes, ou 30 , mais enfin pas mal!

(Frantext: G. Flaubert, Correspondance (I851-juillet I852), Paris, L. Conard, 1926, p. 367)

[18] - [...] Quand on débarque comme ça toute une famille dans un pays qu'on connaît pas, c'est difficile de s'estaller.

- Toute une famille? dit Roumestan, les yeux élargis.

- Bé! oui, le papa, la sœur... on a fait ce que vous disiez.

(Frantext: A. Daudet, Numa Roumestan [188I], Paris, Gallimard, 1994, p. 63)

[19] [...] mes bien-aimés, tous, y compris ma chère petite Dédé. J'espère que son poulet, son pigeon, son chevreau, son chat et son lapin ne l'empêcheront pas d'écrire à son papa. Je lui recommande aussi de bien travailler et d'obéir à sa sœur, qui est grande et sage. Ce qui ne veut pas dire pourtant que Dédé ne soit pas sage.

(Frantext: V. Hugo, Correspondance, Paris, A. Michel - P. Ollendorff, t. I, Lettres à la fiancée (I820-1822). Correspondance (I814-I848), 1947, P. 573)

[20] Il s'appelle Vincent Machot et, même s'il a l'âge de voler de ses propres ailes, il habite toujours chez sa maman.

(Télérama, $\left.\mathrm{n}^{\circ} 3454,2016\right)$

\subsection{Papa, maman, des marqueurs de «proximité communicative »}

Il est sans doute légitime de distinguer deux séries de termes en français, car, même si papa, maman, etc. ne sont pas exclusivement des termes d'adresse et si père, mère, etc. peuvent l'être, ces deux séries se distinguent dans tous leurs emplois par le marquage d'une plus ou moins grande proximité communicative et surtout, parmi les différentes propriétés énumérées par Koch et Oesterreicher (200I) pour

19. Nous reprenons ici l’opposition entre «immédiat» (ou «proximité» chez Gadet, 2008, Riegel et al., 2009) et «distance», opposition «conceptionnelle» entre «parlé» et «écrit» (à distinguer de l'opposition «médiale» entre code phonique et code graphique), du continuum communicatif de Koch et Oesterreicher (200I).

20. Dans un hebdomadaire qui, notamment dans ses résumés et critiques de films, privilégie souvent une certaine proximité avec le lecteur en multipliant les effets humoristiques. 
caractériser proximité et distance communicatives, par une «émotionnalité» plus ou moins forte. Les noms tels que papa, maman, etc. ont en effet une valeur émotionnelle plus forte que père, mère, dans leurs emplois situationnels comme dans leurs emplois anaphoriques; ils contribuent fréquemment à l'expression des sentiments du locuteur. Dans les emplois anaphoriques illustrés plus haut, ils se chargent de valeurs émotionnelles bien précises, affective en [I5] et [19], ironique en $\left[\mathrm{I}_{3}\right],\left[\mathrm{I}_{4}\right]$ et $[\mathrm{I} 7]$, humoristique en $[\mathrm{I} 6]$ et $[20]$.

On retrouve ici certaines des fonctions de la typologie établie par Bartens et Sandström (2006) pour décrire les diminutifs en espagnol et en italien et que Peeters (20I2) évoque pour décrire bien des emplois de petit en français. Petit et papa peuvent d'ailleurs être combinés avec de telles fonctions - affective en [2I], ironique en [22]:

[2I] Que je suis content de ma Didine, mon Adèle! elle a donc une dent, et une dent enfantée sans douleur! Dis-lui bien en l'embrassant mille fois que son petit papa est satisfait de sa conduite en cette occasion, et qu'il portera à sa maman de bons biscuits de Reims qui rendront son lait plus sucré.

(Frantext: V. Hugo, Correspondance, Paris, A. Michel - P. Ollendorff, t. I, Lettres à la fiancée (I820-I822). Correspondance (I8I4-I848), 1947, p. 416)

[22] - [...] Il aurait mieux fait de me dire en face: j'ai envie d'être seul. Parce que c'est ça le fond: il veut pouvoir pleurer tranquillement son petit papa chéri.

- C'est normal, dis-je.

- Normal? Son père était un vieux salaud. D’abord, il n'aurait pas dû se réconcilier avec lui; et maintenant voilà qu'il le pleure.

(Frantext: S. de Beauvoir, Les mandarins, Paris, Gallimard, 1954, p. 338)

Parmi les différentes fonctions associées à l'adjectif petit en français (voir Peeters, 20I2), certaines sont dirigées vers l'interlocuteur et contribuent à l'expression de valeurs illocutoires diverses, témoignage d'affection, reproche, voire insulte (par exemple: «Ouvre tes petits yeux et regarde!», «Vous avez chacun un vélo au cas où vos petites jambes se fatigueraient» [Peeters, 20I2: 1903]), d'autres sont dirigées vers ce dont on parle (par exemple, l'«expression de l'affection ressentie quand on s'adresse à quelqu'un au sujet de quelqu'un d'autre» ou «au sujet de quelque chose» [Peeters, 2012: 1898-1899], qui caractérise les emplois de petit dans bonne petite vie, petit coin tranquille, petite femme, bons petits plats).

En dehors de leurs emplois d'adresse et de leurs emplois "ordinaires» ${ }^{21}$ dans les chaînes de la conversation, papa et maman se chargent des mêmes types de valeurs. Dans leurs emplois situationnels, ils prennent souvent une valeur hypocoristique et sont alors fréquemment combinés avec un déterminant possessif (à un enfant:

21. C'est-à-dire les emplois décrits dans la section 3, qui supposent l'appartenance du locuteur et de son interlocuteur à une communauté épistémique restreinte, à l'intérieur de laquelle papa ou maman représente le «désignateur propre» de l’individu. 
Tu peux aller chercher ton papa ou ta maman?; à quelqu'un dont la mère est malade: Comment va ta maman?), mais ils peuvent aussi prendre une valeur méprisante et renforcer une insulte (C'est ça, va te plaindre auprès de (ta) maman). Dans leurs emplois anaphoriques, les fonctions affectives, ironiques ou humoristiques peuvent s'appliquer aux différents référents impliqués dans la relation, c'est-à-dire soit au référent associé au $\mathrm{SN}$ anaphorique ( $p a p a$, le papa, son papa) soit à celui associé à son antécédent, le «possesseur» ou la famille entière avec les anaphores associatives: l'affection exprimée est celle ressentie pour le "possesseur» en [19] et [2I] - la fille d'Hugo, i. e. le possesseur compris dans le son de son papa, son petit papa-, la cible de l'humour est le «possesseur» en [20] - Vincent, fils à sa maman -, l'ironie est aussi dirigée vers le «possesseur» en [13] et [I4] - Kev Adams, Thomas Fabius -, alors qu'elle est dirigée vers le "possédé», le père, en [22]; enfin l'affection, l'humour ou l'ironie visent la famille entière dans les anaphores associatives de [I5], [I6] et [17].

Plutôt qu'à une variation diaphasique - comme le suggère l'étiquette «familier» souvent attribuée à papa et maman dans les dictionnaires - les emplois de papa, maman vs père, mère semblent ainsi liés à une variation «parlé»/《écrit» au sens conceptionnel de Koch et Oesterreicher (200I): ils marquent une plus grande proximité communicative, en particulier une plus grande «émotionnalité», et contribuent fréquemment à la réalisation d'actes illocutoires «expressifs» (Searle, 1982) impliquant une forte participation émotionnelle du locuteur, témoignages d'affection, mais aussi insultes, moqueries.

\section{Conclusion}

Ces noms, qualifiés de «familiers», «enfantins» ou «affectueux» par les dictionnaires, se distinguent des autres noms de parenté par le marquage d'une plus grande proximité communicative. Se distinguent-ils aussi des autres noms de parenté par leurs propriétés relationnelles? Sont-ils, par exemple, moins fortement relationnels que les autres noms de parenté, parce qu'ils apparaissent plus fréquemment sans marque de dépendance? $\mathrm{Ou}$, au contraire, plus fortement relationnels, parce qu'ils n'ont même plus besoin d'une telle marque de dépendance dans des emplois pourtant strictement relationnels?

Ce n'est probablement pas en ces termes hiérarchiques que la distinction entre papa, maman et les autres noms de parenté doit être posée. Ce qui caractérise papa, maman ou encore fiston ${ }^{22}$ par rapport aux autres noms de parenté, c'est leur

22. Nous ajoutons fiston pour souligner que, si l'on peut distinguer à l'intérieur de la série papa, maman des degrés relationnels comparables à ceux de la série père, mère (papa, maman autorisent comme père, mère les constructions attributives sans déterminant et sans complément relationnel, caractéristiques des noms faiblement relationnels au sens de Barque (2015): Max est papa/père; fiston, comme le nom fortement relationnel fils, n'autorise pas une telle construction: * Max est fiston/fils), ces différences ne paraissent pas avoir d'incidences sur les emplois nus évoqués ici. Fiston connaît en effet, comme papa, maman, des emplois d'adresse, mais aussi des emplois anaphoriques, à interprétation possessive (exemple: «[...] le papa se lève, vérifie que fiston va bien, le rassure et retourne se coucher [...]»; forum du site 
indexicalité ${ }^{23}$, et cela dans leurs emplois strictement relationnels comme dans leurs emplois comme noms de membres. Papa, maman sont des noms indexicaux qui désignent directement l'interlocuteur dans leurs emplois d'adresse et qui peuvent signaler, indépendamment de toute détermination nominale, un lien contextuel, situationnel (lien avec un des protagonistes de la conversation : papa $a$ «mon père», plus rarement «ton père») ou textuel (lien avec un individu ou une collection évoqués dans le contexte antécédent: papa $=$ «le père de $\mathrm{X}$ » ou «le père de la famille $\mathrm{X} »)$. Parce qu'ils ont cette propriété indexicale, leur combinaison avec d'autres marqueurs indexicaux (les déterminants possessifs notamment) est parfois perçue comme redondante; elle accentue en outre les effets expressifs déjà liés à papa, maman, tout particulièrement les effets affectifs et hypocoristiques (avec les SN ton papa, son papa et son petit papa [chez Hugo] adressés à un/son enfant).

Certes, père, mère connaissent des emplois indexicaux similaires ${ }^{24}$. Outre leurs emplois d'adresse, ils peuvent en effet se substituer à papa, maman pour désigner le père ou la mère du locuteur (voir l'exemple [Iza] dans lequel la locutrice utilise Père pour parler de son père) ou, dans des emplois anaphoriques, pour désigner le père d'un tiers : père pourrait remplacer papa dans les emplois nus de [I3] et [I4], si l'on estimait que Kev Adams et Thomas Fabius appellent leur père père et non papa (les emplois de père ou papa dans ces contextes-là sont peut-être des formes de mentions). Mais ces emplois indexicaux paraissent d'une part plus limités que pour la série papa, maman (père, mère semblent difficiles dans les emplois associatifs de [15] et [16]), d'autre part plus ou moins réservés aux noms - faiblement relationnels au sens de Barque (2015) - père, mère, grand-père et grand-mère: fils, fille, frère, mari - fortement relationnels - autorisent difficilement ces emplois nus (sauf, éventuellement, dans quelques emplois d'adresse); oncle, tante - faiblement relationnels - et cousin - fortement relationnel - autorisent les emplois sans déterminant à condition d'être suivis d'un nom propre identifiant (oncle Max, cousine Juditb).

La question des emplois discursifs des noms de parenté est donc loin d'être réglée: il reste, entre autres, à expliquer les différences qu'on vient d'évoquer entre les différents noms de la série père, grand-père, fils, oncle, cousin et à décrire les

«Nanny secours», http://www.nannysecours.com) ou associative (exemple: «Un panneau, au carrefour de la nationale et de la route secondaire, montrait une famille hilare - papa, maman, fiston et la petite frangine - s'avançant au milieu d'un merveilleux pays de manèges, de jeux et de productions agricoles»; Google Books: S. King, Cellulaire, Paris, A. Michel, 2010).

23. Propriété que nous définirons à la suite de Cornish (1999:22), comme la propriété: "of "pointing" to an element of the context which will enable the adressee to identify the intended referent: via the deictic procedure in terms of the utterance or discourse context (see the phenomenon termed "discourse deixis"), and via the anaphoric procedure only in terms of discourse context». Voir aussi Cornish (2015).

24. Contrairement à ce que soutient Takagaki (20I6). Nous n'avons pas repris ici l'étiquette de «noms de titre familial» que Takagaki (20Io et 2016 ) réserve à la série papa, maman, dans la mesure où père, mère sont, eux aussi, susceptibles de constituer des «titres» (dans leurs emplois d'adresse et autres emplois nus). Voir Cornulier (200I: I5) sur les emplois situationnels nus de père, mère. 
particularités des emplois anaphoriques nus des noms de la série papa, pépé, fiston, tata: formes de mentions dans les emplois «possessifs» 25 (exemples [I3]-[I4]) ? Et dans les emplois associatifs (exemples [15]-[16])?

\section{Références}

Aikhenvald, A. Y. 20I3. Possession and Ownership: A Cross-Linguistic Perspective. In A. Y. Aikhenvald et R. M. W. Dixon (éd.), Possession and Ownership. A Cross-Linguistic Typology. Oxford: Oxford University Press: $\mathrm{I}-64$.

Barker, C. 1995. Possessive Descriptions. Stanford: CSLI Publications.

Barker, C. 2oir. Possessives and Relational Nouns. In K. von Heusinger, C. Maienborn et P. Portner (éd.), Semantics. An International Hanbook of Natural Language Meaning. Berlin: De Gruyter Mouton. Vol. 2: Iıo9-IIzo.

BARQue, L. 2015. Les noms relationnels de type humain. Langue française 185:29-4I.

Bartens, A. et SAndström, N. 2006. Towards a Description of Spanish and Italian Diminutives within the NSM Framework. In B. PeEters (éd.), Semantic Primes and Universal Grammar: Empirical Evidence from the Romance Languages. Amsterdam - Philadelphie : J. Benjamins: 33I-36o.

Beyssade, C. 1994. Les modalités épistémiques dans un système multi-agent: contribution à l'étude de l'opacité et des présuppositions. Thèse de doctorat. Université de Caen.

Beyssade, C. 1998. Sens et savoirs: des communautés épistémiques dans le discours. Rennes: Presses universitaires de Rennes.

Charolles, M. 2002. La référence et les expressions référentielles en français. Gap - Paris: Ophrys.

Corblin, F. 2005. Les chaînes de la conversation et les autres. En ligne sur HAL: https:// jeannicod.ccsd.cnrs.fr/ijn_oo54I9I9. I-ı6. [Article publié dans: J.-M. Gouvard (éd.), De la langue au style. Lyon: Presses universitaires de Lyon: 233-254.]

Cornish, F. 1999. Anaphora, Discourse, and Understanding: Evidence from English and French. Oxford: Clarendon Press.

Cornish, F. 20I5. Procédures référentielles indexicales, relations rhétoriques et structuration du discours. Discours I7: I-28. En ligne à l'adresse suivante: http://discours.revues. org/9098.

Cornulier, B. DE 200I. Des réflexifs d'emploi aux noms propres: Je, Bibi, Maman... et quelques autres. In B. DE CORNULIER, Tigres et autres problèmes de sémantique [recueil d'articles publiés par l'auteur sur la sémantique de 1978 à 2000]. Nantes: Centre d'analyse linguistique du discours (CALD), université de Nantes: II-25.

De Bruin, J. et Scha, R. 1988. The Interpretation of Relational Nouns. In J. HobBs (éd.), Proceedings of the 26th Annual Meeting of the Association for Computational Linguistics. Stroudsburg: Association for Computational Linguistics: 25-32. En ligne à l'adresse suivante : https://aclweb.org/anthology/P/P88/P88-Ioo4.pdf.

25. Takagaki (20Io et 20I6) analyse ces emplois nus avec référence à un possesseur de troisième personne comme des marques d'empathie. Il est cependant difficile de parler d'empathie, d'identification du locuteur aux fils à papa que sont Kev Adams et Thomas Fabius, dans les emplois ironiques de papa en [I3] et [I4]. 
GADET, F. 2008. Variation et polygraphie: les écrits électroniques. In C. BRISSAUD, J.-P. JAFFRÉ et J.-C. Pellat (éd.), Nouvelles recherches en orthographe: actes des journées d'études des I4 et I5 juin 2007, université de Strasbourg. Limoges: Lambert-Lucas: 7I-83.

GIRY-SCHNEIDER, J. I99I. L'article zéro dans le lexique-grammaire des noms prédicatifs. Langages 102: 23-35.

Hız, H. 1968. Referentials. Transformations and Discourse Analysis Papers 76. Philadelphie: University of Pennsylvania. En ligne à l'adresse suivante: http://www.cs.nyu.edu/cs/ projects/lsp/pubs/tdap_76_i968.pdf.

HuYghe, R. 2015. Les typologies nominales: présentation. Langue française 185: 5-27.

Joosten, F. 2oro. Collective Nouns, Aggregate Nouns, and Superordinates. When "Part of" and "Kind of" Meet. Lingvistice Investigationes 33 (I) : 25-49.

Kleiber, G. 1999. Les noms relationnels en anaphore associative: le cas des noms de parenté. Studi italiani di linguistica teorica e applicata 28 (2): 283-300.

Kleiber, G. 200o. Le possessif via l'anaphore associative. In M. L. Casal Silva, G. Conde Tarrío, J. Lago Garabatos, L. Pino Serrano et N. Rodríguez Pereira (éd.), La lingüística francesa en España camino del siglo XXI. Madrid: Arrecife. Vol. I : 55-74. En ligne à l'adresse suivante: http://dialnet.unirioja.es/descarga/articulo/4044357.pdf. [Article également publié en 200I dans: L. TAsmowski (éd.), The Expression of Possession in Romance and German Languages. Cluj-Napoca: Editura Clusium : 29-52.]

KLEIBER, G. 200I. L'anaphore associative. Paris: PUF.

KLEIBER, G. 2002. Micro-anaphorique et macro-anaphorique avec une vue spéciale sur les noms de parenté en anaphore associative. In H. L. ANDERSEN et H. NøLKE (éd.), Macro-syntaxe et macro-sémantique: actes du colloque international d'Arbus, I7-I9 mai $200 I$. Berne: P. Lang: 207-238.

KLEIBER, G. 2003. Un «puzzle» référentiel en anaphore associative. In F. I. FonsECA et A. M. Brito (éd.), Lingua portuguesa: estruturas, usos e contrastes. Porto: Centro de Linguística da Universidade do Porto: 97-ıı.

Kleiber, G. 2008. The Semantics and Pragmatics of the Possessive Determiner. In H. H. Müller et A. Klinge (éd.), Essays on Nominal Determination: From Morphology to Discourse Management. Amsterdam - Philadelphie: J. Benjamins: 309-336.

Koch, P. et Oesterreicher, W. 200I. Langage parlé et langage écrit/Gesprochene Sprache und geschriebene Sprache. In G. Holtus, M. Metzelin et C. Schmitt (éd.), Lexikon der romanistischen Linguistik. Tübingen: M. Niemeyer. Vol. I, 2: Methodologie: Sprache in der Gesellschaft; Sprache und Klassifikation; Datensammlung und -verarbeitung: 584-627.

Kupferman, L. I99i. Structure événementielle de l'alternance un/ $\varnothing$ devant les noms humains attributs. Langages I02: 52-75.

Lecolle, M. 1998. Noms collectifs et méronymie. Cabiers de grammaire 23:4I-65.

Milner, J.-C. 1982. Ordres et raisons de langue. Paris: Seuil.

Nakamura, H. et Mori, Y. 2004. Relational Nouns as Anaphors. In H. Masuichi, T. Ohкuma, K. Ishikawa, Y. Harada et K. Yoshimoto (éd.), Proceedings of the I8th Pacific Asia Conference on Language, Information and Computation - PACLIC I8. Stroudsburg: Association for Computational Linguistics: 7I-80. En ligne à l'adresse suivante: https:// www.aclweb.org/anthology/Y/Yo4/Yo4-I008.pdf. 
PeEters, B. 20I2. Les petites idées d'un petit Belge, ou quand petit ne renvoie pas à la taille. In F. Neveu, V. Muni Toke, P. Blumenthal, T. Klingler, P. Ligas, S. Prévost et S. Teston-Bonnard (éd.), SHS Web of Conferences. Actes $d u 3^{e}$ congrès mondial de Linguistique française - CMLF 2012 (Lyon, 4-7 juillet 20I2). Les Ulis: EDP Sciences. Vol. I : i893-1907. En ligne à l'adresse suivante: https://www.shs-conferences.org/ articles/shsconf/pdf/20I2/oI/shsconf_cmlfi2_0ooo7I.pdf.

Riegel, M., Pellat, J.-C. et Rioul, R. 2009. Grammaire méthodique du français. Paris: PUF [ 4 éd.].

SEARle, J. R. 1982. Sens et expression: études de théorie des actes de langage. Paris: Minuit.

TAKAGAKI, Y. 20IO. Les noms de titre familial comme marqueurs de cohérence. In F. NEVEU, V. Muni Toke, J. Durand, T. Klingler, L. Mondada et S. Prévost (éd.), Actes $d u 2^{e}$ congrès mondial de Linguistique française - CMLF 2010 (La Nouvelle-Orléans, I2-I5 juillet 20IO). Paris: Institut de linguistique française: I215-I225. En ligne à l'adresse suivante: https://www.linguistiquefrancaise.org/articles/cmlf/pdf/20ro/or/ cmlf2OIO_000253.pdf.

TAKAGAKI, Y. 20I6. Les interprétations des noms de titre familial en emploi non vocatif: l'embrayeur, l'empathie et les plans d'énonciation. In F. Neveu, G. Bergounioux, M.-H. Côté, J.-M. Fournier, L. Hriba et S. PrÉvost (éd.), SHS Web of Conferences. Actes du $5^{e}$ congrès mondial de Linguistique française - CMLF 20I6 (Tours, 4-8juillet 20I6). Les Ulis: EDP Sciences. Vol. 27: I-I3. En ligne à l'adresse suivante: https://www. shs-conferences.org/articles/shsconf/pdf/2016/05/shsconf_cmlf2016_06009.pdf.

VILlar, M. B. 2006. Quel est le rôle de la possession inaliénable dans la description de la relation lexicale partie-tout? In G. Kleiber, C. Schnedecker et A. Theissen (éd.), La relation partie-tout. Louvain - Paris: Peeters : $367-379$.

Wilmet, M. 20i6. Ces noms communément appelés propres et improprement appelés communs. Langue française $190: 15-28$.

\section{Base de données}

ATILF (laboratoire Analyse et traitement informatique de la langue française). Base textuelle Frantext. En ligne à l'adresse suivante: http://www.frantext.fr/. 